\title{
Adsorption of Naphthalene and Pyrene Containing Surfactant Peptides onto Single- Walled Carbon Nanotubes: A Microscopy, Spectroscopy, and Theoretical Study
}

\author{
D.R. Samarajeewa, R.J.K.U. Ranatunga, B.A. Wilson, A.T. Lemieux, G.R. Dieckmann, S.O. Nielsen, \\ and I.H. Musselman
}

Department of Chemistry, The University of Texas at Dallas, 800 West Campbell Road, Richardson, TX 75080

We examined the interaction between polycyclic aromatic hydrocarbons (PAHs) and single-walled carbon nanotubes (SWCNTs) using both experiments and molecular dynamics (MD) simulations. PAH molecules have a hexagonal arrangement of $\mathrm{sp}^{2}$-hybridized carbon atoms, the same structural motif shared by carbon nanomaterials such as graphene and carbon nanotubes. Therefore, PAHs form $\pi-\pi$ stacking interactions with other graphitic surfaces and can be used as dispersal agents for SWCNTs [1].

In the current work, we attached PAH molecules onto the $N$-terminus of short, amphiphilic peptides referred to as surfactant peptides (SP) [2]. The structure of each peptide consists of two lysine residues at the $C$-terminus, forming a polar head, followed by five valine residues and an $N$-terminal aromatic residue, forming an apolar tail. The hydrophobic effect, together with $\pi-\pi$ interactions of the aromatic moiety with the nanotube surface, drives the apolar segment of the peptide to adsorb onto the SWCNT surface, while the polar segment facilitates the dispersion of SWCNTs in aqueous media. Two surfactant peptides, namely SP-na and SP-py, were synthesized by incorporating naphthalene (na), which has two fused aromatic rings, and pyrene (py) with four fused aromatic rings, as the $N$-terminal aromatic moiety. A peptide synthesized by incorporating a single phenyl ring (SP-ph) was used as the reference.

SP-PAH/SWCNT dispersions were prepared by probe sonication and ultracentrifugation [3]. The SPna/SWCNT dispersion showed the darkest color, suggesting that SP-na is able to create a higher concentration of dispersed SWCNTs in water under the experimental conditions used. Diluted dispersions were used for atomic force microscopy (AFM). The coverage of surfactant peptides on SWCNTs was studied using a Multimode ${ }^{\circledR} 8$ Scanning Probe Microscope operated in peak force QNM ${ }^{\mathrm{TM}}$ (quantitative nanomechanical mapping) under ambient conditions. Compared to SP-ph, both SP-na and SP-py appear to provide greater peptide coverage on the SWCNTs (Figure 1). Figure 2 shows AFM images of the peptide/SWCNT dispersions as well as peptide control samples acquired using TappingMode ${ }^{\mathrm{TM}}$. AFM height measurements of SWCNTs were performed on regions devoid of peptide. The resultant average diameters were close to $1 \mathrm{~nm}$ (number of SWCNTs $=20$ from each sample), suggesting that most of the SWCNTs are singly dispersed. Both SP-ph/SWCNT and SPna/SWCNT composites show a small amount of particulate matter in the sample backgrounds, whereas a relatively large amount of excess material was observed for the SP-py/SWCNT composite. AFM studies performed on the peptide controls clearly show the presence of large tubular peptide aggregates for the SP-py sample indicating its ability to self-aggregate.

MD simulations performed to examine the aggregation behavior of the surfactant peptides in the absence of SWCNTs are consistent with the AFM images. In Figure 3, snapshots obtained from systems containing 60 SP-PAH molecules show that the peptide aggregate size increases with increasing number of PAH rings. The interaction between SP-na and SP-py molecules is largely governed by $\pi-\pi$ stacking 
interactions, with SP-py molecules showing a greater ability to associate than SP-na molecules. This result may explain why SP-py is slightly less effective in dispersing SWCNTs than SP-na.

Scanning tunneling spectroscopy (STS) studies were performed using a NanoScope ${ }^{\circledR}$ V STM under ambient conditions. STS I-V curves were acquired from peptide-coated SWNTs (bias voltage range from -1.0 to $1.0 \mathrm{~V})$ and were numerically converted to differential conductance $(\mathrm{dI} / \mathrm{dV})$ spectra in order to study the electronic properties of the SWNTs. Most of the STS dI/dV spectra acquired from the peptide-coated SWCNTs did not show additional peaks near the Fermi level or any significant conduction or valence band peak shifting (data not shown). This confirms that the studied aromatic rings do not cause significant alterations to the SWCNT electronic structure.

[1] S. Debnath et al., J. Phys. Chem. C 114 (2010), p. 8167.

[2] H. Xie et al., J. Pept. Sci. 14 (2008), p. 139.

[3] D.R. Samarajeewa et al., Carbon (2013), http://dx.doi.org/10.1016/j.carbon.2013.01.039.

(a)
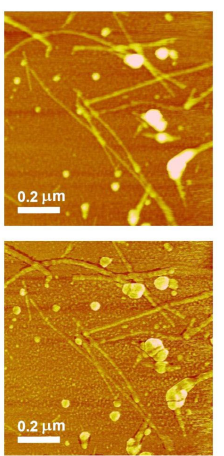

(b)
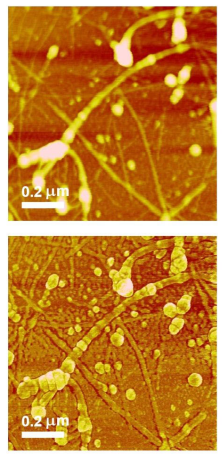

(c)

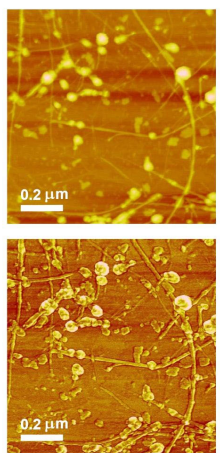

(d)

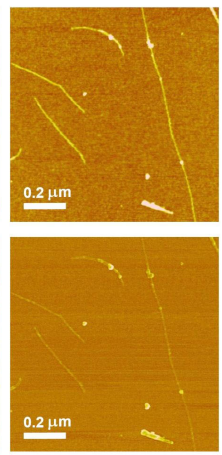

Figure 1. Peak Force QNM ${ }^{\mathrm{TM}}$ height images (top) and deformation maps (bottom) of (a) SPph/SWCNT, (b) SP-na/SWCNT, (c) SP-py/SWCNT, and (d) bare SWCNT. Peptide/SWCNT dispersions were diluted 20-fold.
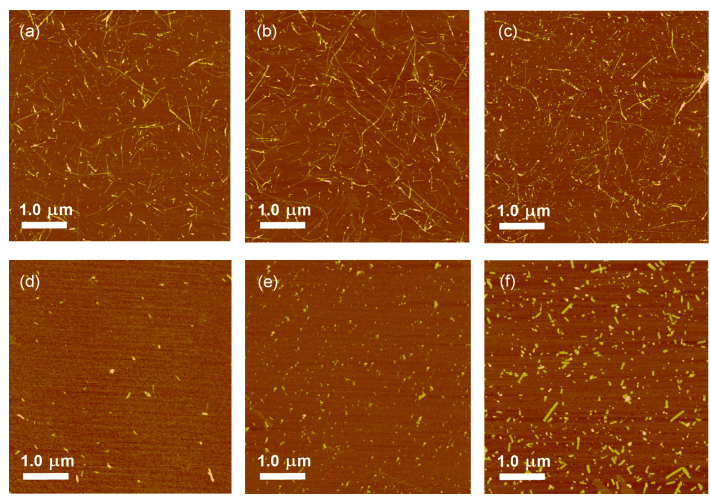

Figure 2. AFM images of peptide/SWCNT dispersions (top) and peptide solutions (bottom). (a) SPph/SWCNT, (b) SP-na/SWCNT, (c) SP-py/SWCNT, (d) SP-ph, (e) SP-na, and (f) SP-py. SP$\mathrm{ph} / \mathrm{SWCNT}$ and SP-ph solutions were diluted 20-fold. SP-na/SWCNT, SP-py/SWCNT, SP-na, and SP-py solutions were diluted 40-fold.
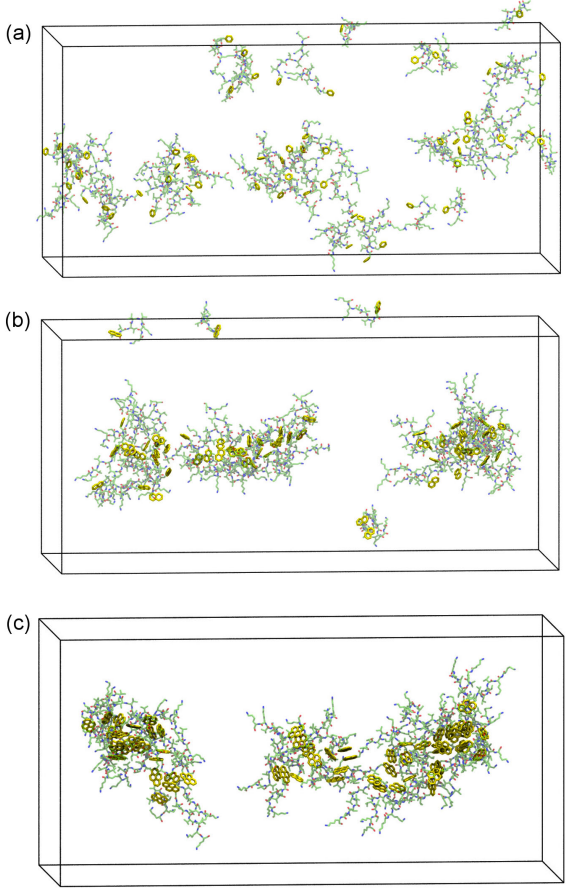

Figure 3. (a) SP-ph/SWCNT, (b) SPna/SWCNT, and (c) SP-py/SWCNT. Hydrogen atoms and water molecules are not shown. $N$-terminal PAHs are highlighted for clarity. 